\title{
Original
}

\section{Biopsia de masas renales: resultados y seguridad en 2009}

\author{
José M. de la Morena Gallego, Carlos Llorente Abarca
}

Hospital Fundación Alcorcón. Madrid. España.

\section{Resumen}

El papel de la biopsia de masas renales ha sido hasta ahora limitado por los resultados insatisfactorios en cuanto a fiabilidad diagnostica y por su escasa repercusión en las decisiones terapéuticas. Recientemente las BMR han recobrado interés entre los urólogos, debido al aumento de incidencia de tumores renales, la aparición de nuevas opciones de manejo y los avances en el diagnóstico histológico.

Hemos revisado mediante una búsqueda en Pubmed, las publicaciones más relevantes sobre biopsias de tumores renales aparecidas en los últimos años.

Los estudios clínicos más recientes demuestran un alto grado de fiabilidad diagnostica para diferenciar malignidad, tipo histológico y grado de las biopsias percutáneas de tumores renales, con una baja tasa de complicaciones asociadas. Sin embargo su utilización rutinaria continúa siendo controvertida y sus indicaciones limitadas dada la escasa capacidad de modificar la actitud terapéutica.

Palabras clave: Cáncer renal. Biopsia. Diagnóstico.

\section{Renal mass biopsy: Results and safety in 2009}

\section{Abstract}

The role of renal mass biopsies has been limited by suboptimal results in terms of diagnostic reliability, and their scant repercussion upon therapeutic decision taking. Recently, however, renal mass biopsies have generated renewed interest among urologists, as a result of the increased incidence of renal tumors, the introduction of new management options, and advances in histological diagnosis.

A review has been made, based on a PubMed search of the most relevant publications on renal tumor biopsies in recent years.

The most recent clinical studies reveal a high degree of diagnostic reliability in differentiating malignancy, histological type and grade in percutaneous biopsies of renal tumors, with a low rate of associated complications. However, routine use of the technique remains controversial, and its indications limited, due to the scant capacity of the renal biopsy findings to modify treatment decision.

Keywords: Renal cell carcinoma. Biopsy. Diagnosis.

$\mathrm{E}$ los últimos años se ha producido un aumento en el interés por la biopsia de masas renales (BMR). El aumento en la incidencia de cáncer renal y especialmente de tumores de pequeño tamaño, consecuencia del incremento en la utilización de pruebas de imagen como el CT y RM ${ }^{1-2}$ es probablemente uno de los motivos, pero también lo es la ampliación en los últimos años de las opciones de manejo de masas renales pequeñas, como las técnicas de ablación térmica o la observación.

El hallazgo incidental de masas renales de pequeño tamaño se ha convertido en un escenario clínico frecuente y en la actualidad se sabe que corresponde con un grupo heterogéneo de tumores que incluyen enfermedades malignas y benignas con comportamiento clínico diferente. Desde una perspectiva tradicional, las biopsias de masas renales presentaban una alta tasa de falsos negativos. Las limitaciones de la fiabilidad diagnóstica de las biopsias de masas renales se ha relacionado con la heterogeneidad histológica de los tumores renales, o con errores en la elección del sitio de punción (errores de muestreo) ${ }^{3-6}$. Pero también por el temor a las potenciales complicaciones o el asiento de células malignas en el sitio de punción ${ }^{7-12}$.

Los urólogos han considerado hasta hace poco candidatas a cirugía todas las lesiones sólidas renales. Sin embargo recientes estudios que han revisado los resultados histopatológicos de piezas de nefrectomía parcial por tumores de pequeño tamaño han demostrado que la mayoría de las lesiones renales de pequeño tamaño presentan bajo grado histológico o características de escasa agresividad y por tanto podrían manejarse en determinados casos sin necesidad de cirugía ${ }^{13,14}$.

En la actualidad, existen nuevos tratamientos dirigidos específicamente hacia dianas moleculares implicadas en el desarrollo de determinados subtipos 
histológicos de Cáncer Renal. Por tanto, el conocimiento de estos subtipos es ahora más necesario a la hora de planear el tratamiento adecuado cuando el tumor se presenta en estadio avanzado o diseminado. Al mismo tiempo, los patólogos disponen de nuevos marcadores moleculares e inmunohistoquímicos para mejorar la fiabilidad de los resultados anatomopatológicos ${ }^{15}$.

A continuación revisaremos los aspectos técnicos, complicaciones y resultados de las BMR en los estudios clínicos más recientes.

\section{ASPECTOS TÉCNICOS DE LA BIOPSIA DE MASAS RENALES}

Hay varios aspectos que repercuten en la precisión y exactitud de las biopsias y que dependen directamente de la técnica empleada.

\section{Aspectos previos a la biopsia}

En general, como para cualquier procedimiento invasivo, los pacientes deben ser estudiados para descartar problemas de coagulación no conocidos o bien ser advertidos de la necesidad de discontinuar el tratamiento con fármacos antiagregantes. Los pacientes anticoagulados deben ser revertidos adecuadamente los días previos y mantenidos con heparinas hasta el día anterior a la punción.

Además, es necesaria una revisión cuidadosa de las imágenes disponibles para elegir el acceso más adecuado a la lesión en cada caso. La inexistencia de una vía segura de punción a la masa es una relativa contraindicación para la biopsia con aguja ${ }^{16}$.

\section{Elección de la imagen guia}

Los estudios más recientes con punción percutánea de masas renales han demostrado no tener mayor numero de biopsias fallidas o indeterminadas que los estudios con biopsia bajo visión directa en quirófano o exvivo.

En general, en series recientes, la mayoría realiza las biopsias bajo control con CT y/o Eco. La MRI es menos frecuentemente usada debido a la necesidad de utilizar agujas de material no ferromagnético. No obstante, ningún estudio ha demostrado la superioridad de una de ellas. La elección, por tanto, debe basarse en la situación clínica, localización de la lesión, características del paciente y preferencias personales del profesional encargado de hacer la biopsia.

\section{Elección de la Aguja de Biopsia}

En la actualidad es asumido que las agujas de biopsia 18 gauge, en general, pueden extraer suficiente tejido para analizar. Agujas 20 g o más pequeñas pueden ser útiles en casos determinados con mayor riesgo de sangrado o cercanía de vísceras en el trayecto de punción. Sin embargo, se ha indicado que las agujas $18 \mathrm{~g}$ o mayores proveen mayor exactitud en el análisis del tejido con poco o ningún incremento en las complicaciones ${ }^{17}$.

\section{4.-Aspectos relativos a la punción}

Parte de las dificultades de exactitud de las biopsias se deben a errores de muestreo. Se recomiendan al menos dos pases de punción, que son en general suficientes para obtener tejido adecuado. Igualmente, un mayor numero de pases no se ha relacionado definitivamente con más complicaciones.

En estudios clínicos se ha comunicado insuficiente material para estudio en el 0-22\% de los casos. Esto representa un error en el lugar de punción en esas lesiones. Es importante obtener muestras de varias áreas del tumor debido a la presencia frecuente de áreas de hemorragia o necrosis central. El tamaño del tumor es probablemente un factor importante a la hora de analizar los resultados. Los tumores mayores son más propensos a la necrosis central mientras que los de tamaño menor presentan mayor dificultad para localizar exactamente la aguja.

\section{COMPLICACIONES}

Las complicaciones potenciales de la biopsia incluyen el sangrado, infección, neumotórax o fístula arteriovenosa. Sin embargo en las series más recientes la incidencia de complicaciones con consecuencias clínicas es muy poco frecuente. La implantación de células tumorales en el sitio de punción ha sido descrita tradicionalmente como una potencial limitación a la biopsia, aunque el riesgo estimado es menor de un 0,01\% y hasta el momento solo 6 casos han sido publicados ${ }^{7-12}$ (Tabla 1).

Las complicaciones se han clasificado en menores y mayores en función de la necesidad de tratamiento, estancia en el hospital y consecuencias derivadas. Las series más largas de biopsias renales comunican pocas complicaciones menores (5\%) y ninguna complicación mayor. Las más frecuentes son: 
Tabla 1. Casos publicados hasta la fecha por implantación tumoral en sitio de punción

\begin{tabular}{lccc}
\hline & Aguja (gauge) & Tiempo hasta presentación & Hallazgos patologicos \\
\hline Gibbons et al & 18 & $20 \mathrm{Mos}$ & RCC \\
Auvert et al & Not available & $84 \mathrm{Mos}$ & Oncocytoma \\
Kiser et al & 14 & $24 \mathrm{Days}$ & Papillary RCC \\
Wehle and Grabstald & 20 & $48 \mathrm{Mos}$ & RCC \\
Shenoy et al & 23 & $12 \mathrm{Mos}$ & RCC \\
Abe and Saitoh & 14 & $18 \mathrm{Mos}$ & Liposarcoma \\
\hline
\end{tabular}

Adaptado de: A. Volpe, J. R. Kachura, W. R. Geddie, A. J. Evans, A. Gharajeh, A. Saravanan and M.A.S. Jewett Techniques, Safety and Accuracy of Sampling of Renal Tumors by Fine Needle Aspiration and Core Biopsy. J Urol 2007 Aug;178(2):379-386 ${ }^{16}$.

\section{Sangrado}

Debido a la naturaleza extremadamente vascularizada del parénquima renal y en general de los tumores renales, es habitual que la biopsia produzca sangrado intratumoral y perinéfrico. Como norma habitual los pacientes deben permanecer de 1 a 3 horas en el hospital tras el procedimiento y si se advierten signos como cambio en las constantes vitales, o dolor intenso deben realizarse las pruebas de imagen pertinentes. En las series más recientes no se han documentado hematomas clínicamente significativos. Ralls et al. realizaron CT o ECO rutinariamente posterior al procedimiento. El 85-91\% de ellos presentaron cierto grado de sangrado visible en las imágenes, aunque las necesidades de transfusión o estancia hospitalaria solo fue del 1-2 \% Más recientemente, Lechevalier en un estudio similar encontró hematomas de diferente grado en el $44 \%$ de los casos. Ninguno preciso ampliar su estancia hospitalaria ni otras medidas terapéuti$\operatorname{cas}^{19}$

\section{Otras complicaciones son infrecuentes}

La infección es rara y fácilmente tratable. Las fístulas arteriovenosas ocurren en pocas ocasiones y han de ser sospechadas en casos de sangrado persistente. El tratamiento mediante embolización es efectivo en casi todos los casos. El neumotórax ocurre con mayor probabilidad cuando se accede posteriormente para punción de masas en polo superior, especialmente si la vía de acceso es entre la 11 y 12 costilla. En esta situación entre un 14 y un 29\% de los casos pueden desarrollar neumotórax. No obstante los casos de neumotórax clínicamente relevantes son muy infrecuentes.

Por otro lado no existe evidencia que demuestre que la biopsia con aguja pueda complicar una posterior cirugía ya sea nefrectomía radical o parcial.

\section{INDICACIONES DE BIOPSIA DE MASAS RENALES}

La BMR tiene sus indicaciones más establecidas en casos en los que el resultado puede evitar la cirugía. Desde un punto de vista clásico, la demostración de masas renales en el seno de una enfermedad diseminada de dudoso origen primario, linfoma o sospecha de absceso precisan de la realización de una punción biopsia.

Cualquier tipo de cáncer puede metastatizar en el riñón. En algunas series entre el 8\% y el $13 \%$ de las masas renales corresponden a metástasis siendo los más frecuentes en cáncer de Pulmón, Colon, Melanoma y el Hepatocarcinoma ${ }^{20,21}$. Por otro lado, la presentación de un masa renal irresecable o asociada con metástasis o la sospecha de Linfoma puede precisar para su correcto manejo, una caracterización histológica mediante punción biopsia.

\section{RESULTADOS Y LIMITACIONES}

Los resultados de los estudios clínicos iniciales sobre biopsia de masas renales concluyeron con un bajo valor predictivo negativo. Los factores más importantes que contribuyeron a la falta de exactitud de las biopsias con aguja fueron los errores de punción y la conocida heterogenicidad en la histología de las masas renales. Resulta especialmente difícil para el patólogo la diferenciación entre el Carcinoma de Células Renales con citoplasma granular o las variantes eosinofílicas del CCR cromófobo del oncocitoma, cuando se cuenta con una cantidad de material para estudio tan escaso. Por otro lado, los tumores renales presentan áreas necróticas o hemorrágicas en muchas ocasiones. La punción profunda o inadecuada puede generar material no valido para el análisis. Estas biopsias no deberían ser catalogadas como falsos negativos, sino como biopsias fallidas o insuficientes para el diagnostico. 
En general, los tumores pequeños producen más errores por dificultad de identificación y punción mientras que en tumores más grandes el problema es localizar la zona de tumor sin necrosis. En una serie de 115 biopsias con aguja se produjo error de punción en el $2,3 \%$ de los tumores con tamaño entre 4 y $6 \mathrm{~cm}$ mientras que en tumores entre 1 y 3 $\mathrm{cm}$ o mayores de $6 \mathrm{~cm}$ este mismo error se produjo en el 13 y $12 \%$ respectivamente ${ }^{22}$. Lechevalier comunicó tasas de falsos negativos del 37\% en biopsias de tumores menores de $3 \mathrm{~cm}$ mientras que en tumores mayores solo fue del $9 \%{ }^{19}$.

En una revisión sobre biopsia de masas renales se analizaron los datos de estudios publicados antes de 2001 y posteriormente ${ }^{23}$. Se recogieron 28 estudios de biopsia de masas renales con 2.474 pacientes en total antes de 2001. En estos estudios los falsos negativos fueron entre el 0 y el $25 \%$. Aunque muchos de estos contenían tejido necrótico, hemorrágico o riñón normal, por lo que deberían clasificarse más adecuadamente como biopsia insuficiente o fallida. En un análisis conjunto de los datos de estos estudios se produjo error de punción en el $8,1 \%$. El patólogo no fue capaz de caracterizar la lesión en el 5,6 \% y los falsos positivos y negativos fueron, respectivamente, el 1,3 y $4 \%$. Por tanto, en un $17,7 \%$ de las biopsias $(8,1+5,6+4 \%)$ los resultados fueron negativos y la aproximación diagnostica de la biopsia en conjunto corresponde en estos estudios al $81,9 \%$.
En la actualidad sabemos que el $20 \%$ de las lesiones renales de pequeño tamaño son tumores benignos. Por otro lado el $60 \%$ de los malignos tienen en realidad un comportamiento indolente y pueden manejarse potencialmente con técnicas de ablación térmica o en algunos casos incluso con observación ${ }^{24}$. Pero ¿cual es la realidad en cuanto a las biopsias de masas renales en 2009? Los resultados de las series más recientes publicadas se resumen en la Tabla 2. En general la mayoría de los autores utiliza aguja 18 gauge y se excluyen las biopsias de lesiones quísticas.

La sensibilidad y especificidad de la biopsia con aguja de masas renales es en series recientes del $70-100 \%$ y $100 \%$ respectivamente, con una exactitud del 90\%.

En 26 biopsias percutáneas utilizando aguja 18 gauge, Caoli et al. obtuvieron un 100\% de sensibilidad y especificidad ${ }^{25}$.

Lechevalier et al. observaron un $89 \%$ de exactitud diagnostica para malignidad y un $78 \%$ para el tipo histológico en una serie de 73 biopsias. El mismo grupo ha comunicado más recientemente un $92 \%$ de exactitud diagnostica para malignidad y tipo histológico en 88 biopsias de masas menores de 4 $\mathrm{cm}^{19}$.

Más recientemente, Volpe et al. obtuvieron un $84 \%$ de biopsias suficientes para el diagnostico. Además, en su estudio fue posible precisar el subtipo histológico y el grado en el 93\% y el 68\% respec-

Tabla 2

\begin{tabular}{|c|c|c|c|c|c|c|}
\hline & No. & Imagen & Aguja (gauge) & $\begin{array}{l}\text { \% Biopsias no } \\
\text { diagnosticas }\end{array}$ & $\begin{array}{c}\text { No. Biopsias } \\
\text { malignas/ } \\
\text { No. confirmadas }\end{array}$ & \% Resultados \\
\hline Wood et al & 79 & CT/US & 22 (PAAF), 17-20 (cores) & 6.3 & $49 / 41$ & Sensibilidad 93, exactitud 95 \\
\hline Lechevallier et al & 73 & $\mathrm{CT}$ & 18 & 21 & $48 / 26$ & $\begin{array}{l}\text { Concordancia biopsia + AP } \\
\text { definitiva } 89\end{array}$ \\
\hline Hara et al & 33 & $\mathrm{CT} / \mathrm{US}$ & 18 & 0 & $21 / 15$ & $\begin{array}{l}\text { Concordancia biopsia }+ \text { AP } \\
\text { defzinitiva } 86,7\end{array}$ \\
\hline Caoili et al & 26 & US & 18 & 0 & $19 / 4$ & Sensibilidad + especificidad 100 \\
\hline Harisinghani et al & $28 \dagger$ & $\mathrm{CT}$ & 22 (PAAF), 18 (cores) & 0 & $17 / 16$ & $\begin{array}{l}\text { Concordancia biopsia + AP } \\
\text { definitiva } 100\end{array}$ \\
\hline Neuzillet et al & 88 & $\mathrm{CT}$ & 18 & 9.1 & $66 / 62$ & Fiabilidad 92 \\
\hline Eshed et al & 22 & $\mathrm{CT}$ & 18 & 4.5 & $15 / 14$ & Sensibilidad 93, especificidad 100 \\
\hline Shah et al & 66 & CT/US & 18 & 21 & $37 / 15$ & Exactitud 98 \\
\hline
\end{tabular}

$\dagger$ Incluye solo biopsias de quistes Bosniak III.

Adaptado de A. Volpe, J.R. Kachura, W.R. Geddie, A.J. Evans, A. Gharajeh, A. Saravanan and M.A.S. Jewett Techniques, Safety and Accuracy of Sampling of Renal Tumors by Fine Needle Aspiration and Core Biopsy.J Urol 2007 Aug;178(2):379-86. (16). 
tivamente de los canceres detectados. La concordancia histopatológica fue del $100 \%$ en los individuos operados ${ }^{26}$.

En un estudio similar, Wang et al. han comunicado los resultados de 110 biopsias por masas renales de menos de $4 \mathrm{~cm}$. Con una media de 4 cilindros de biopsia, el 90,9\% fueron suficientes para el diagnostico y el 9,1\% insuficiente o indeterminadas. El $35 \%$ de las biopsias con suficiente tejido fueron interpretadas como benignas. En todos los casos que fueron sometidos a cirugía posteriormente (34 casos) no hubo diferencias en el diagnostico histopatológico. Hubo 8 casos que precisaron tratamiento en urgencias u hospitalización. De ellos 2 fueron hematomas asociados a hipotensión. No obstante todos fueron manejados conservadoramente sin consecuencias. Los autores destacan la fiabilidad de la biopsia y recomiendan su utilización en casos seleccionados de pacientes malos candidatos a cirugía. Sin embargo, se trata de un estudio retrospectivo, en el que los individuos están ya probablemente seleccionados y por tanto la extrapolación de sus resultados debe hacerse con cautela.

En una reciente revisión, Lane et al. analizaron los datos de 7 estudios clínicos posteriores al 2001 con un total de 362 pacientes. El 5,2\% y el 3,8\% de las biopsias fueron insuficientes o indeterminadas respectivamente. En el $0,6 \%$ se produjeron falsos negativos y las complicaciones mayores fueron el $0,3 \%{ }^{23}$.

Algunos autores complementan la biopsia con punción aspiración con aguja fina (PAAF). Los resultados de aproximación diagnostica para la PAAF son peores que los relativos a biopsia y se han publicado resultados con una alta tasa de falsos negativos y positivos. Sin embargo otros grupos comunican buenos resultados y atribuyen esta diferencia a la falta de experiencia en la interpretación de las muestras $^{27,28}$.

La PAAF y biopsia con aguja parecen resultar complementarios. En el caso concreto de biopsias de masas quísticas esto parece ser especialmente cierto según algunos autores. Richter et al. han caracterizado con Biopsia y PAAF combinadas el 89,4\% de 227 quistes complejos Bosniak II/III ${ }^{29}$. En la misma línea Lechevaliier et al. concluye que la fiabilidad de la biopsia con aguja de quistes complejos no es inferior a la obtenida en masas sólidas. No obstante, cuando existen lesiones quísticas bien caracterizadas las decisiones terapéuticas están bien definidas por la clasificación de Bosniak. En las lesiones quísticas la probabilidad de error de punción es mayor que en lesiones sólidas y hay un pequeño pero real riesgo de rotura del quiste y eventualmente de diseminación maligna.

En conclusión, actualmente las biopsias renales tienen un alto grado de exactitud diagnostica cuando se obtiene tejido adecuado para su análisis. Este análisis puede diferenciar igualmente el subtipo histológico y facilitar en determinados casos las decisiones terapéuticas. Sin embargo su utilización rutinaria no esta comúnmente aceptada y las indicaciones deben seguir limitándose a lesiones en las que se sospeche un origen distinto del renal o bien en casos en los que se decida tratar mediante ablación percutánea, en el momento de su realización.

\section{REFERENCIAS}

1. Chow WH, Devesa SS, Warren JL, Fraumeni JF Jr. Rising incidence of renal cell cancer in the United States. JAMA 1999;281(17): 1628-1631.

2. Jemal A, Siegel R, Ward E, Murray T, Xu J, Smigal C et al. Cancer statistics, 2006, CA Cancer J Clin. 2006;56(2):106-130.

3. Wood BJ, Khan MA, McGovern F, Harisinghani M, Hahn PF, Mueller PR. Imaging guided biopsy of renal masses: indications, accuracy and impact on clinical management. J Urol 1999;161(5):1470-1474.

4. Juul N, Torp-Pedersen S, Gronvall S, Holm HH, Koch F, Larsen S. Ultrasonically guided fine needle aspiration biopsy of renal masses. J Urol 1985;133(4):579-81.

5. Niceforo J, Coughlin BF. Diagnosis of renal cell carcinoma: value of fine-needle aspiration cytology in patients with metastases or contraindications to nephrectomy. AJR Am J Roentgenol. 1993;161(6):1303-1305.

6. Murphy WM, Zambroni BR, Emerson LD, Moinuddin S, Lee LH Aspiration biopsy of the kidney. Simultaneous collection of cytologic and histologic specimens. Cancer 1985;56(1):200-205.

7. Gibbons RP, Bush WH Jr, Burnett LL. Needle tract seeding following aspiration of renal cell carcinoma. 1977;118(5):865-867.

8. Kiser GC, Totonchy M, Barry JM., Needle tract seeding after percutaneous renal adenocarcinoma aspiration. 1986;136(6): 1292-1293.

9. Shenoy PD, Lakhkar BN, Ghosh MK, Patil UD. Cutaneous seeding of renal carcinoma by Chiba needle aspiration biopsy. Case report. Acta Radiol. 1991;32(1):50-52.

10. Wehle MJ, Grabstald H. Contraindications to needle aspiration of a solid renal mass: tumor dissemination by renal needle aspiration. J Urol 1986;136(2):446-448.

11. Auvert J, Abbou CC, Lavarenne V. Needle tract seeding following puncture of renal oncocytoma. Prog Clin Biol Res. 1982; 100:597-598.

12. Abe M, Saitoh M. Selective renal tumour biopsy under ultrasonic guidance. Br J Urol. 1992;70(1):7-11.

13. Frank I, Blute ML, Cheville JC, Lohse CM, Weaver AL, Zincke H. Solid renal tumors: an analysis of pathological features related to tumor size J Urol. 2003;170(6 Pt 1):2217-2220.

14. Lane BR, Babineau D, Kattan MW, Novick AC, Gill IS, Zhou M, et al. A preoperative prognostic nomogram for solid enhancing renal tumors $7 \mathrm{~cm}$ or less amenable to partial nephrectomy. J Urol. 2007;178(2):429-434. 
15. Yang XJ, Sugimura J, Schafernak KT, Tretiakova MS, Han M, Vogelzang NJ, et al. Classification of renal neoplasms based on molecular signaturas. J Urol. 2006;175(6):2302-2306

16. Volpe A, Kachura JR, Geddie WR, Evans AJ, Gharajeh A, Saravanan A et al. Techniques, safety and accuracy of sampling of renal tumors by fine needle aspiration and core biopsy. J Urol. 2007;178(2):379-386.

17. Neuzillet Y, Lechevallier E, Andre M, Daniel L, Coulange C. Accuracy and clinical role of fine needle percutaneous biopsy with computerized tomography guidance of small (less than 4.0 cm) renal masses. J Urol. 2004;171(5):1802-1805.

18. Ralls PW, Barakos JA, Kaptein EM, Friedman PE, Fouladian G, Boswell WD et al. Renal biopsy-related hemorrhage: frequency and comparison of $\mathrm{CT}$ and sonography. J Comput Assist Tomogr. 1987;11(6):1031-1034.

19. Lechevallier E, André M, Barriol D, Daniel L, Eghazarian C, De Fromont $\mathrm{M}$ et al. Fine-needle percutaneous biopsy of renal masses with helical CT guidance. Radiology. 2000;216(2):506-510.

20. Bracken RB, Chica G, Johnson DE, Luna M. Secondary renal neoplasms: an autopsy study. South Med J. 1979;72(7):806807.

21. Gattuso P, Ramzy I, Truong LD, Lankford KL, Green L, Kluskens $\mathrm{L}$, et al., Utilization of fine-needle aspiration in the diagnosis of metastatic tumors to the kidney, Diagn Cytopathol. 1999;21(1): 35-38.

22. Rybicki FJ, Shu KM, Cibas ES, Fielding JR, vanSonnenberg E, Silverman SG. Percutaneous biopsy of renal masses: sensitivity and negative predictive value stratified by clinical setting and size of masses, AJR Am J Roentgenol. 2003;180(5):1281-1287.

23. Lane BR, Samplaski MK, Herts BR, Zhou M, Novick AC, Campbell SC. Renal mass biopsy-a renaissance? J Urol 2008; 179(1):20-27.
24. Chawla SN, Crispen PL, Hanlon AL, Greenberg RE, Chen DY, Uzzo RG. The natural history of observed enhancing renal masses: meta-analysis and review of the world literature. J Urol. 2006;175(2):425-431.

25. Caoili EM, Bude RO, Higgins EJ, Hoff DL, Nghiem HV Evaluation of sonographically guided percutaneous core biopsy of renal masses, AJR Am J Roentgenol 179 (2002), p. 37.

26. Volpe A, Mattar K, Finelli A, Kachura JR, Evans AJ, Geddie WR et al. Contemporary results of percutaneous biopsy of 100 small renal masses: a single center experience. J Urol. 2008; 180(6):2333-2337.

27. Renshaw AA, Cibas ES, Kidney and adrenal gland. In: Cibas ES, Ducatman BS, Editors, Cytology: Diagnostic Principles and Clinical Correlates, Saunders Ltd, Edinburgh 2003;383-404.

28. Truong LD, Todd TD, Dhurandhar B, Ramzy I Fine-needle aspiration of renal masses in adults: analysis of results and diagnostic problems in 108 cases. Diagn Cytopathol. 1999;20(6): 339-349

29. Richter F, Kasabian NG, Irwin RJ Jr, Watson RA, Lang EK. Accuracy of diagnosis by guided biopsy of renal mass lesions classified indeterminate by imaging studies. Urology 2000;55(3):348-352.

Correspondencia autor: Dr. José M. de la Morena Gallego Servicio de Urología

Fundación Hospital Alcorcón

Budapest, 1 - 28922 Alcorcón, Madrid

Tel.: 916219400

E-mail autor: jmmorena@hotmail.com

Información artículo: Original 